\title{
BMJ Open Trajectory research in children on the autism spectrum: a scoping review protocol
}

To cite: Gentles S, Duku E, Kerns C, et al. Trajectory research in children on the autism spectrum: a scoping review protocol. BMJ Open 2021;11:e053443. doi:10.1136/ bmjopen-2021-053443

- Prepublication history for this paper is available online. To view these files, please visit the journal online (http://dx.doi org/10.1136/bmjopen-2021053443).

Received 13 May 2021 Accepted 30 October 2021

Check for updates

(C) Author(s) (or their employer(s)) 2021. Re-use permitted under CC BY-NC. No commercial re-use. See rights and permissions. Published by BMJ.

${ }^{1}$ Department of Psychiatry and Behavioural Neurosciences, McMaster University, Hamilton, Ontario, Canada

${ }^{2}$ Department of Psychology, The University of British Columbia, Vancouver, British Columbia, Canada

${ }^{3}$ Michael G. DeGroote School of Medicine, McMaster University, Hamilton, Ontario, Canada

${ }^{4}$ Health Sciences Library,

McMaster University, Hamilton, Ontario, Canada

${ }^{5}$ Department of Psychiatry, University of Toronto, Toronto,

Ontario, Canada

${ }^{6}$ Centre for Addiction and Mental Health, Toronto, Ontario, Canada

Correspondence to

Dr Stephen Gentles;

stevegentles@gmail.com

\section{ABSTRACT}

Introduction Longitudinal trajectory methods, featuring outcome assessments at three or more time points, are increasingly being used as appropriate approaches to understand developmental pathways of people on the autism spectrum across the lifespan. Understanding the scope of this rapidly expanding body of research can help inform future trajectory studies and identify areas for potential meta-analysis as well as key evidence gaps. We present the protocol for a scoping review whose objective is to identify and summarise the scope of research that uses a longitudinal trajectory study design to examine development in children diagnosed with autism. Specifically, we will identify outcome domains and age intervals that have been well characterised, areas where further research is needed and the historical use of various longitudinal trajectory analytical approaches.

Methods and analysis We outline the methods for the proposed scoping review according to the framework outlined by Arksey and O'Malley, with subsequent clarifications and enhancements by other authors. Using a search strategy developed by a medical librarian, we will search six databases for relevant publications. Titles and abstracts will be screened in duplicate, followed by full-text screening. Data extraction fields developed predominantly a priori from a set of guiding subquestions will be used to chart relevant data. The findings will include quantitative aggregate summaries, narrative summaries, and appraisal of trajectory studies according to our methodological subquestions. We will consult autistic self-advocate and parent-caregiver stakeholders to facilitate interpretation of the findings.

Ethics and dissemination Research ethics approval is not required for this scoping review. The results will be presented to researcher, care professional, policy-maker and stakeholder audiences at local and international conferences, other dissemination activities and published in a peer-reviewed journal.

\section{BACKGROUND}

Autism (autism spectrum disorder) is a neurodevelopmental condition recently estimated to affect 1 in 54 children, ${ }^{1}$ the prevalence of which is not likely to be substantively affected by geography, race or socioeconomic factors. ${ }^{2}$ The diagnosis
Strengths and limitations of this study

- The scoping review whose protocol is presented will be the first to establish which outcome domains and age intervals have been characterised by longitudinal trajectory research examining development in children diagnosed with autism, and which warrant further study.

- An innovative aspect of this scoping review will be its use of prespecified subquestions to guide development of the extraction (charting) form.

- We will summarise information corresponding to methodological subquestions that may provide a useful basis for future critical appraisal of trajectory studies in this area.

- This review is limited in its scope because it excludes trajectory studies whose focus is on adulthood, and a separate review is therefore warranted to report on trajectories of outcome domains that may only be relevant at later life stages.

is defined by variation in social communication and interaction, and restricted, repetitive patterns of behaviour, interests or activities. ${ }^{3}$ Autism is also increasingly being understood to be characterised by different strengths. ${ }^{4}$ It has long been recognised that the characteristics used to define autism vary developmentally over the life-course, and that there is a need for rigorous longitudinal research to understand changes in relevant outcomes over time, identify prognostic factors and understand what may improve developmental pathways of relevant outcome domains. ${ }^{5}$

Trajectory methodology, defined here as featuring longitudinal analysis of assessments at three or more time points, has emerged as more appropriate for understanding development in autism compared with traditional cohort studies characterised by assessments at only two time points. In an early review of longitudinal research in autism that included studies with traditional 
cohort and cross-sectional designs, Seltzer et at highlighted the key limitations of research featuring assessments at only two time points, noting it 'makes it impossible to characterise the shape of the developmental function, the timing of changes, or the possibility that there are different subtypes of individuals on the autism spectrum characterised by different trajectories to the same outcome.' Recognising these limitations, researchers in the field of autism have increasingly turned to trajectory methods, which feature assessments at three or more time points. Some large-scale longitudinal autism cohort research groups have published serial reports on the development of their child cohorts, increasing the number of assessments as they age over time. ${ }^{78}$ Such research has expanded our understanding of autism in important ways, including by defining important variation in the developmental trajectories of children on the autism spectrum-variation that exists both between trajectory groups or clusters, and between children within those clusters (for example see, $\left.{ }^{9-11}\right)$. Consequently, other approaches, which simply report or graph average measures across individuals at each time point, have limited use for studying development in people on the autism spectrum, because they ignore and obscure the correlated nature of within-case data over time.

Given the expansion of published trajectory studies in autism, there is a need to understand its scope to provide a broad picture of aspects such as the variety of outcome domains (ie, measurable outcomes, which potentially vary over development) that have been studied over time, the age intervals over which outcome domains have been followed, and trends in the statistical analytic approaches that have been used. Two broad types of analytic approaches have been used to study autism trajectories: multilevel modelling (MLM), a variable-centred approach that estimates the average intercept and slope of the outcome domain of interest for predefined cohort groups (eg, autistic, non-autistic); and growth mixture modelling (GMM), a personcentred approach that estimates distinct trajectories of latent groups formed on the basis of similar trajectories of individual participants within a cohort population.

\section{Previous reviews}

The early reviews on longitudinal studies in autism (published 2004-2013) of which we are aware neglected to report a systematic search strategy, and have at least some focus on adults on the autism spectrum. ${ }^{5612}$ Three subsequent reviews of longitudinal autism research (published 2014 onwards), which did report a systematic search strategy, included studies following up to adulthood. Magiati et $a l^{13}$ included 25 studies and summarised findings from multiple outcome domains including cognitive ability, language, adaptive functioning, autism severity and social functioning; a focus of this review was childhood predictors of later outcomes, and a mix of studies using trajectory and traditional two-time point cohort designs were included but not separated from each other in the review. Bieleninik et $a l^{14}$ conducted a systematic review and meta-analysis that included 35 prospective cohort studies and 5 randomised controlled trials, and evaluated two outcome domains: diagnostic stability, and autism symptom severity; trajectory studies were not distinguished from two-time point follow-up studies. Howlin and Magiati ${ }^{15}$ reviewed all adult outcome-focused research (43 studies), of which five were trajectory studies described individually in a separate section. A search for existing published (PubMed) or registered (PROSPERO) protocols did not reveal other scoping reviews of trajectory studies in autism research.

Our planned scoping study will be the first we are aware of to review research that uses a longitudinal trajectory study design to study change in outcome domains over time in children on the autism spectrum (to age 18). Notably, it will exclude studies whose focus is on adulthood (ie, where at least half of age time points assessed are above 18 years), because the set of outcome domains that are relevant after the transition to adulthood differs sufficiently from childhood, in our view, to warrant a separate review. Namely, some domains relevant to early development (eg, language) are generally less relevant in adulthood, while numerous adultrelevant domains are inapplicable to early childhood (eg, employment status, romantic relationships). The planned review will address the breadth of outcome domains relevant to children on the autism spectrum including clinical (developmental, behavioural, functional), educational (eg, academic achievement) and social. We will, however, exclude studies of trajectories of neuroanatomical or physiological development, which have been previously reviewed. ${ }^{16} 17$

\section{Objectives}

The primary objective of this scoping review is to identify and summarise the scope of research that uses a longitudinal trajectory study design (with three or more time points) to study the progression of different outcome domains-including the shape, timing and subgroups-in children on the autism spectrum (to age 18 ). A secondary objective is to summarise methodological trends in terms of analytic approaches used in trajectory studies of child development in autism research. Findings from this review will provide a useful understanding of (1) how and where trajectory research has already been used, and the areas where future trajectory research can produce needed knowledge about autism (gaps), (2) specific outcome domains or research questions for which sufficient data exist to conduct more focused quantitative systematic reviews or meta-analyses and (3) the general utility and value of trajectory study methodology for providing actionable knowledge to support positive development of children on the autism spectrum. 


\section{METHODS AND ANALYSIS}

The scoping review methodology was judged to be appropriate for this review topic given its breadth, objectives, and the lack of known recent reviews on similar topics. ${ }^{18}$ We outline the methods here according to the scoping review framework put forth by Arksey and O'Malley, ${ }^{19}$ informed by subsequent clarifications and enhancements, ${ }^{20}{ }^{21}$ reporting relevant elements outlined in the corresponding PRISMA Extension for Scoping Reviews. ${ }^{22}$

\section{Stage 1: identifying the research question}

The primary objective and corresponding research question for this review was informed by the experience of review group members with trajectory studies of children on the autism spectrum ${ }^{72324}$ and by an examination of existing reviews on the topic which identified three narrative reviews in children ${ }^{61617}$ and five additional reviews of trajectories to adulthood. ${ }^{512-15}$ In addition to helping confirm the appropriateness and need for our focus on children, the examination of existing reviews helped us understand the potential scope of the literature and, later, to iteratively exclude two research categories we felt represented distinct topics of interest warranting separate reviews: (1) studies of trajectories of neuroanatomical or physiological development and (2) descriptive case studies or case series of individual trajectories. The two primary research questions for this review that we used to inform study identification and selection are as follows:

- How has research that employs a longitudinal trajectory study design (ie, featuring three or more time points and an aggregative statistical analysis that accounts for the correlated nature of within-case data over time) been used to produce knowledge about the change-including shape, timing and subgroups-in child developmental, behavioural, functional, social or outcome domains in children on the autism spectrum (to age 18)?

-What are some of the key methodological characteristics of this research?

The first question above identifies the key domains of our search strategy and screening criteria, namely the population (diagnosis and age), methodology and outcome domains measured (ie, non-anatomical). Under the umbrella of the primary research questions, lead investigators on this review (SGen, ED and SGeo) also developed a set of subquestions (box 1) that were used to plan the data extraction and reporting of results, described below.

\section{Stage 2: identifying relevant studies}

Our search was informed by the domains of the primary research question described above, and developed with the help of a medical librarian (LB), starting with Medline (OVID) because the subject heading definitions of this database are well developed (box 2).

The MEDLINE search was translated into the five other databases to be searched: EMBASE, CINAHL, PsycINFO, ERIC, Cochrane Database of Systematic Reviews. Search

\section{Box 1 Subquestions to guide data extraction}

\section{Questions about scope of the research}

1. What outcome domains have been studied in trajectory studies?

2. What outcome measures have been used to follow the different outcome domains over time?

3. What ages have been characterised for each outcome domain?

4. For studies using person-centred (growth mixture modelling, GMM) approaches, how many trajectory groups (or clusters) have been defined for different outcome domains followed in different trajectory studies?

5. What and where are the different autism cohort research groups that have reported trajectory study findings?

6. To what extent have each of the different autism cohort research groups contributed to the trajectory study literature, and what are the general characteristics of their research (narrative summary)?

7. To what extent have the proposed implications (utility) of trajectory study findings been reported clearly?

8. How much impact (in terms of citation metrics) have different sources or types of trajectory research had?

\section{Questions about methodological aspects of research}

1. What terms (text words) have been used to describe multilevel modelling (MLM) and GMM trajectory studies of child development in autism?

2. How has the historical prevalence of MLM-type and GMM-type trajectory approaches for studying development of children on the autism spectrum evolved by year?

3. How do select study characteristics (eg, sample size, comparison to non-autism groups) vary between MLM-type and GMM-type studies?

4. How reliable is the ascertainment of autism diagnosis in trajectory studies?

5. To what extent was representative sampling used across trajectory studies?

6. What other methodological aspects of trajectory research in autism could be used as a basis for identifying sources of risk of bias or for quality reporting standards?

results will be imported into EndNote reference management software (Clarivate Analytics), where duplicates will be removed and citations managed in the subsequent screening stages. We will not search the grey literature or conference proceedings, because we are interested in mapping successfully peer-reviewed published literature that is likely to influence the field. For similar reasons, we will exclude doctoral theses or dissertations retrieved by the searches. Searches will not be restricted by language or year of publication. We will include articles published from database inception to the year 2020, based on a search completed 24 May 2021. The expected study completion date is December 2021.

\section{Stage 3: study selection}

After removal of duplicates from our EndNote database, we will select articles for inclusion in the review through a two-step screening process: title and abstract, and fulltext screening. In both steps, all records will be screened in duplicate (by two reviewers), and disagreements will be resolved through consensus in regular meetings. 


\section{Box 2 Medline search strategy}

1. exp Autism Spectrum Disorder/

2. Child Development Disorders, Pervasive/

3. Asperger*.mp.

4. ASD.mp.

5. autis*.mp.

6. or/ $1-5$

7. exp Child/

8. child ${ }^{\star} . \mathrm{mp}$.

9. Adolescent/

10. Infant/

11. Infant, Newborn/

12. infan*.mp.

13. newborn*.mp.

14. Child, Preschool/

15. Child Development/

16. adolescen*.mp.

17. Pediatrics/

18. youth.mp.

19. teen*.mp.

20. p?ediatric*.mp.

21. or $/ 7-20$

22. trajector*.mp.

23. Longitudinal Studies/

24. Follow-Up Studies/

25. Prospective Studies/

26. Iongitudinal ${ }^{*} . \mathrm{mp}$.

27. follow-up stud*.mp.

28. prospective*.mp.

29. follow-up stud ${ }^{\star}$.mp.

30. Cohort Studies/

31. (cohort* stud $^{*}$ or cohort analys?s).mp.

32. panel stud*.mp.

33. or $/ 22-32$

34. 6 and 21 and 33

Reviewer decisions will be recorded in EndNote, with mutual consensus required before an include or exclude decision is reached. For the first step, title and abstract screening, screening decisions will err on the side of inclusivity (sensitivity), so that records for which insufficient information is available in the title or abstract will be included, and passed on, to the second screening step. Based on preliminary work, in this step we expect to partially filter records based on indicators of diagnosis (autism), age and methodology (trajectory design), and definitively exclude records based on their identity as case studies or series, trajectories of neuroanatomy or physiology, or non-published status (eg, dissertations or theses).

In the second step, the full text of articles will be retrieved in Portable Document Format (PDF) and read to confirm eligibility. Based on preliminary work, one of the predominant decisions in this step will be to determine if studies qualify as true trajectory study methodology both in terms of the data collection criterion (three time points or more) and two analysis criteria (analysis accounts for the correlated nature of within-case data
Table 1 Information fields for extraction from each included publication

\begin{tabular}{|c|c|}
\hline Category & Information extraction field \\
\hline $\begin{array}{l}\text { Article } \\
\text { characteristics }\end{array}$ & $\begin{array}{l}\text { Year of publication } \\
\text { Article impact } \\
\text { Country(ies) of origin of cohort } \\
\text { Purpose of trajectory study (narrative) } \\
\text { Applicability of findings (narrative) } \\
\text { Clarity of applicability of findings }\end{array}$ \\
\hline $\begin{array}{l}\text { Cohort research } \\
\text { group }\end{array}$ & $\begin{array}{l}\text { Autism cohort research group: title } \\
\text { Autism cohort research group: lead } \\
\text { author }\end{array}$ \\
\hline Sample & $\begin{array}{l}\text { Sample setting (community based, } \\
\text { clinical, etc) } \\
\text { Type of sampling of autism participants } \\
\text { (non-representative, representative) } \\
\text { Sample size, overall } \\
\text { Sample size, autism only }\end{array}$ \\
\hline $\begin{array}{l}\text { Diagnostic } \\
\text { ascertainment }\end{array}$ & $\begin{array}{l}\text { Autism diagnosis methods reported? } \\
\text { Use of autism diagnostic observation } \\
\text { schedule } \\
\text { Use of autism diagnostic interview- } \\
\text { revised } \\
\text { Clinical judgement used }\end{array}$ \\
\hline Analysis & $\begin{array}{l}\text { Prospective } \\
\text { MLM type } \\
\text { MLM terms used } \\
\text { GMM type } \\
\text { GMM terms used } \\
\text { Clarity of ages of assessment }\end{array}$ \\
\hline $\begin{array}{l}\text { Outcome domain } \\
\text { (repeated for each } \\
\text { domain) }\end{array}$ & $\begin{array}{l}\text { Outcome domain name } \\
\text { Measure used } \\
\text { Age interval start } \\
\text { Age interval end } \\
\text { No of time points assessed } \\
\text { Ages of time points assessed } \\
\text { Rationale for ages assessed (narrative) } \\
\text { Clinical schedule used } \\
\text { No of trajectory groups (clusters) defined }\end{array}$ \\
\hline
\end{tabular}

GMM, growth mixture modelling; MLM, multilevel modelling.

over time; and analysis does not simply average measures across individuals at each time point yielding only crosssectional estimates). Given the potential number and complexity of analytic techniques, a statistician will be involved as a second reviewer for full-text screening decisions involving analysis-related criteria where necessary. Reasons for exclusions at the full-text screening step will be recorded in EndNote. A Preferred Reporting Items for Systematic Reviews and Meta-Analyses (PRISMA) flow chart will be used to report the results of the searches and both screening steps.

\section{Stage 4: charting the data}

The fields that we will extract data on (table 1) have been developed as much as possible, a priori, from the subquestions listed in box 1 . These fields are captured in an initial extraction form designed in Word, with the input of lead investigators (SGen, ED and SGeo) and the extraction team, to streamline the extraction process (eg, containing 
in-line instructions and other needed reference information). This form was piloted by the four-person extraction team (SGen, MCH, AJM and ECNC) who each independently applied it to one paper for training and reliability purposes. The form was judged easy to use by all, there was little disagreement, and misunderstandings were discussed and corrected. We anticipate it will be possible to develop a relatively stable form for addressing the prespecified subquestions. Nevertheless, we expect some unanticipated but important extraction fields will become apparent in the course of reviewing the included literature, which may require the iterative adaptation of the extraction form. Consequently, we have included a field in the extraction form to record ideas or suggestions for adaptation or revision that may iteratively arise when reviewing a specific article.

Information regarding methodological quality of individual studies will be extracted to address two of our subquestions. Some extraction fields pertaining to trajectory methodology will be developed iteratively as there are no existing methodological quality criteria that we are aware of for this study design, and it is not possible to anticipate all aspects of quality in advance of reviewing this literature. Additionally, quality indicators already accepted in the field of epidemiological autism research will be extracted, namely those pertaining to researchlevel diagnostic ascertainment standards.

Given the inevitability that there will be multiple publications by the same study group on the same cohort as it is reported on over multiple age time points, it may be possible to find helpful information pertaining to a given study in other publications by the same cohort research group. In addition to keeping careful records of which publications are linked in this way (as an extraction and reporting field of interest), we will assign papers belonging to the same cohort group to the same extractor so they can be aware of, and cross-reference as needed, the shared context of linked papers while extracting.

While articles will be extracted individually, we will have regular extraction meetings to discuss challenging articles, ideas for revising the extraction form, and other issues. Additionally, extractors will be able to consult with each other regarding areas of uncertainty related to specific publications. Finally, completed extraction forms will be verified by the review coordinator (SGen) before transferring their data to the analysis database, maintained in Excel, which will allow for aggregative summary and cross-tabulation of the extracted data. Fields in the Excel form will be programmed with data validation settings to minimise errors in data entry.

\section{Stage 5: collating, summarising and reporting the results}

Along with presenting standard publication retrieval in a PRISMA flow chart, we plan to report on the number of unique studies that were captured by each successive database, from MEDLINE, to Embase, PsycINFO, CINAHL, ERIC, and Cochrane Reviews.
We expect a high number of included studies $(>60)$, which may affect the level of aggregation we employ to summarise data presentations. We plan to present a summary table displaying key characteristics of the extracted literature, organised hierarchically by country, major cohort research group (where applicable) and individual publication (as hierarchically indented rows); and include citations for each of the characteristics presented in columns-including sample sizes, outcome domains followed, age interval followed, number of time points, analytic approach and citation impact. We also plan to use graphs to visually represent data on historical trends in the use of MLM versus GMM analytic approaches over time, and on the density of age time point assessments for each outcome domain. In addition, we will present data that may provide a useful basis for future critical appraisal of trajectory studies according to our methodological subquestions regarding autism diagnosis ascertainment, and trajectory review method reporting criteria. A table will display a proposed set of text words that can be used to search for each type of trajectory analysis approach (MLM and GMM) in future.

A section of the review will provide narrative summaries to address the subquestion regarding the impact and extent to which the major autism cohort research groups have contributed to the trajectory study literature including general characteristics of their research. Narrative will also be used to provide interpretive commentary on other subquestion-related aspects, including suitability of the outcome measures that have been used to follow the different outcome domains over time (including their patient-centredness, per stakeholder consultation described below), the number of trajectory groups (clusters) that have been defined for different outcome domains, the extent to which the implications (or utility) of trajectory study findings have been reported clearly, and the merit of future research to target gaps in the ages or outcome domains not previously assessed.

\section{Patient and public involvement}

Corresponding to stage 6 of the Arkseyand O'Malley scoping review framework (optional consultation exercise), we have engaged two stakeholders in this reviewan adult Autistic self-advocate and a parent of a child on the autism spectrum-to provide feedback at key points. For purposes of this protocol, they have provided early feedback on aspects of the planned extraction related to outcome domains. This feedback informed plans for interpreting the relevance and importance of different outcomes in the final report, but it did not alter our decision to extract and report on all outcomes assessed by the studies to be included in this review. We did not involve patient stakeholders in development of the research question, or design of this study. While they will not be involved in data collection or analysis, we will re-engage these individuals once the review is complete to provide interpretations of the findings from their perspectives as members of the stakeholder community. Their interpretations will 
be used to inform the discussion of findings. We will also involve these individuals in presentations of the results described in the next section, according to their interest.

\section{ETHICS AND DISSEMINATION}

The research ethics boards at our institutions do not need to be engaged to provide ethics approval for consulting with stakeholders about this research project since it does not involve their providing study data. We will follow best practices for patient engagement in research.

This review will represent a source of valuable knowledge and guidance to researchers who are currently engaged in, or planning to conduct, longitudinal research on child development in autistic samples. It will also be of interest to clinicians, policy-makers and other professionals responsible for care or services for children on the autism spectrum, allowing them to quickly identify the trajectory literature on clinically relevant outcome domains. It will also be of interest to families of children on the autism spectrum, who are known for their desire for access to research findings, by providing an overview of the kind of research that is often conducted to find answers about 'what to expect' during the development of children on the autism spectrum. Finally, it will be useful to other reviewers seeking to identify one or more outcome domains for which there is sufficient published trajectory research data to conduct a systematic review or meta-analysis. To reach these audiences, we plan to disseminate findings at major international autism research conferences, local autism conferences attended by families, and to publish the completed review in open access format in a peer-reviewed autism research journal. Other dissemination activities include plans to develop a webinar for parent and professional audiences, and lay research summaries for publication on provincial and national autism organisation web sites.

\section{Twitter Stephen Gentles @SteveGentles}

Acknowledgements The authors would also like to thank Trudy Goold and Connie Putterman for providing feedback from a stakeholder perspective on aspects of the proposed data extraction.

Contributors SGen, SGeo and ED led the design and conceptualisation. EDB, LB and SGeo developed the search strategy. AJM, ECN-C, MCH, CK and PS were involved in design and conceptualisation of the extraction form. SGeo drafted the protocol. SGen, ED, AJM, ECN-C, MCH, CK and PS provided feedback and helped revise drafts of this manuscript for important intellectual content and clarity.

Funding The authors have not declared a specific grant for this research from any funding agency in the public, commercial or not-for-profit sectors.

\section{Competing interests None declared.}

Patient and public involvement Patients and/or the public were involved in the design, or conduct, or reporting, or dissemination plans of this research. Refer to the Methods section for further details.

Patient consent for publication Not applicable.

Provenance and peer review Not commissioned; externally peer reviewed.

Open access This is an open access article distributed in accordance with the Creative Commons Attribution Non Commercial (CC BY-NC 4.0) license, which permits others to distribute, remix, adapt, build upon this work non-commercially, and license their derivative works on different terms, provided the original work is properly cited, appropriate credit is given, any changes made indicated, and the use is non-commercial. See: http://creativecommons.org/licenses/by-nc/4.0/.

\section{ORCID iD}

Stephen Gentles http://orcid.org/0000-0003-2004-1451

\section{REFERENCES}

1 Maenner MJ, Shaw KA, Baio J, et al. Prevalence of autism spectrum disorder among children aged 8 years - autism and developmental disabilities monitoring network, 11 sites, United States, 2016. MMWR Surveill Summ 2020;69:1-12.

2 Elsabbagh M, Divan G, Koh Y-J, et al. Global prevalence of autism and other pervasive developmental disorders. Autism Res 2012;5:160-79.

3 Association, A.P. Diagnostic and statistical manual of mental disorders. 5 edn. American Psychiatric Association, 2013: 1-36.

4 Mahdi S, Albertowski K, Almodayfer O, et al. An international clinical study of ability and disability in autism spectrum disorder using the WHO-ICF framework. J Autism Dev Disord 2018;48:2148-63.

5 Howlin P, Moss P. Adults with autism spectrum disorders. Can J Psychiatry 2012;57:275-83.

6 Seltzer MM, Shattuck P, Abbeduto L, et al. Trajectory of development in adolescents and adults with autism. Ment Retard Dev Disabil Res Rev 2004;10:234-47.

7 Szatmari P, Georgiades S, Duku E, et al. Developmental trajectories of symptom severity and adaptive functioning in an inception cohort of preschool children with autism spectrum disorder. JAMA Psychiatry 2015;72:276-83.

8 Gotham K, Pickles A, Lord C. Trajectories of autism severity in children using standardized ADOS scores. Pediatrics 2012;130:e1278-84.

9 Georgiades S, Bishop SL, Frazier T. Editorial Perspective: Longitudinal research in autism - introducing the concept of 'chronogeneity'. J Child Psychol Psychiatry 2017;58:634-6.

10 Charman T. Mapping early symptom trajectories in autism spectrum disorder: lessons and challenges for clinical practice and science. $J$ Am Acad Child Adolesc Psychiatry 2018;57:820-1.

$11 \mathrm{Kim} \mathrm{SH}$, Bal VH, Benrey N, et al. Variability in autism symptom trajectories using repeated observations from 14 to 36 months of age. J Am Acad Child Adolesc Psychiatry 2018;57:837-48.

12 Henninger NA, Taylor JL. Outcomes in adults with autism spectrum disorders: a historical perspective. Autism 2013;17:103-16.

13 Magiati I, Tay XW, Howlin P. Cognitive, language, social and behavioural outcomes in adults with autism spectrum disorders: a systematic review of longitudinal follow-up studies in adulthood. Clin Psychol Rev 2014;34:73-86.

14 Bieleninik Łucja, Posserud M-B, Geretsegger M, et al. Tracing the temporal stability of autism spectrum diagnosis and severity as measured by the autism diagnostic observation schedule: a systematic review and meta-analysis. PLoS One 2017;12:e0183160.

15 Howlin P, Magiati I. Autism spectrum disorder: outcomes in adulthood. Curr Opin Psychiatry 2017;30:69-76.

16 Baribeau DA, Anagnostou E. A comparison of neuroimaging findings in childhood onset schizophrenia and autism spectrum disorder: a review of the literature. Front Psychiatry 2013;4:175.

17 Lainhart JE. Brain imaging research in autism spectrum disorders: in search of neuropathology and health across the lifespan. Curr Opin Psychiatry 2015;28:76-82.

18 Pollock D, Davies EL, Peters MDJ, et al. Undertaking a scoping review: a practical guide for nursing and midwifery students, clinicians, researchers, and academics. J Adv Nurs 2021;77:2102-13.

19 Arksey H, O'Malley L. Scoping studies: towards a methodological framework. Int J Soc Res Methodol 2005;8:19-32.

20 Levac D, Colquhoun H, O'Brien KK. Scoping studies: advancing the methodology. Implement Sci 2010;5:69.

21 O'Brien KK, Colquhoun H, Levac D. Advancing scoping study methodology: a web-based survey and consultation of perceptions on terminology, definition and methodological steps. BMC Health Services Research 2016;16:1-12.

22 Tricco AC, Lillie E, Zarin W, et al. PRISMA extension for scoping reviews (PRISMA-ScR): checklist and explanation. Ann Intern Med 2018;169:467-73.

23 Szatmari P, Bryson S, Duku E, et al. Similar developmental trajectories in autism and Asperger syndrome: from early childhood to adolescence. J Child Psychol Psychiatry 2009;50:1459-67.

24 Di Rezze B, Duku E, Szatmari P, et al. Examining trajectories of daily living skills over the preschool years for children with autism spectrum disorder. J Autism Dev Disord 2019;49:4390-9. 